\title{
Consistent Multipath Dynamic Source Routing Protocol for Mobile Ad hoc Network
}

\author{
N. M. Lutimath, S. L and C. Naikodi
}

\begin{abstract}
Mobile Ad hoc Network consists of a large number of mobile nodes that communicate with each other without any infra-structure. Utilizing efficient energy of the nodes and selection of routes from the primary and secondary caches plays an important role. We propose a consistent multipath dynamic source routing protocol (CM-DSR) for mobile ad hoc network by selecting consistent route from cache. The results are simulated using NS-2 simulator. The observations show that our proposed protocol performs better in selection of routes in terms of average residual energy, average transmission per unit time for given density of nodes in the topology, which increases the data transmission from each node to other nodes in the network.
\end{abstract}

Index Terms-DSR, Multipath, Residual Energy, Transmission Energy.

\section{INTRODUCTION}

With the improvement in mobile computing devices the requirement for wireless communication without fixed infrastructure has engrossed great interest in mobile ad hoc networks. A Mobile Ad hoc Network (MANET) is infrastructure-less, adaptive wireless communication network that requires no fixed radio base stations. Each mobile node acts as host as well as router which is capable of routing and moving independently. There is either direct wireless link or multi-hop wireless link between each node in the communication network. Examples of ad hoc network applications are in defense, where soldiers relaying information for situational awareness during war, business stake holders sharing information through meetings, and disaster relief personnel managing efforts after natural disaster.

MANETs protocols can mainly group into two types. They are the proactive and reactive [1] or on demand protocols. Proactive or table driven protocols transmit periodic control packets. Up-to-date routing information is stored in packets from each node to all nodes in the network. A typical proactive protocol is DSDV [2]. Reactive protocols maintain update routing information at each node in an on-demand fashion. Reactive protocols attempt to discover a path to the destination only when a node initiates a packet for sending towards to that destination. Typical reactive protocols are DSR [3] and AODV [4].When a

Manuscript published July 31, 2016

Nagaraj M. Lutimath, Dept. of CSE, SVCE, Bangalore, India. (e-mail: nagarajlutimath@gmail.com)

Suresh L, Dept. of CSE, Cambridge Institute of Tech., Bangalore, India. (e-mail: suriakls@gmail.com)

Chandrakant Naikodi, Dept. of CSE, Cambridge Institute of Tech., Bangalore, India. (e-mail: nadhachandra@gmail.com) reactive protocol originates a route; the route discovery procedure basically involves network-wide query broadcasting. To minimize the overhead and the delay of initiating a route discovery mechanism, reactive protocols need to cache the routes previously discovered in the route cache of each node. Because of frequent movement of nodes the cached routes are liable to become stale. This is due to the lack of timely update mechanisms to the route cache. The stale cached routes would cause packet loss and increase delivery latency and delay. As a result, a route cache with no effective update mechanism may considerably reduce system performance [5].

The main goal of our work is to design a consistent protocol to be able to continue transmit the important information in a MANET. A collection of wireless communication network with each node varying battery power is taken for study. We propose a consistent dynamic source routing protocol which uses the routes in both primary cache and secondary cache. A consistent route is found using the routes found in primary and secondary caches taking residual energy of the route. By examining residual energy and transmission energy of the given source and destination nodes at a given internal of time our proposed protocol performs better than typical dynamic source routing protocol (DSR). The rest of our paper is organized into following sections. Section II describes related works. Section III defines the problem. Section IV the proposed work is explained. Section V simulation results with performance evaluation is done. Section VI lastly concludes the paper.

\section{RELATED WORKS}

Considerable research have been taken place in the area of mobile ad hoc networks, where new protocols have been designed and utilized to improve efficiency of classical protocols. Some of the related works are described below.

A Minimum Energy Routing (MER) [6] that finds a route that consumes the minimum amount of energy to get the packet to the destination is described by authors. The protocol requires the cost of a link in terms of the energy consumed to successfully transfer and receive data packet over the link

MER suffers from higher routing overhead, a minimum total energy, and works within a range of static and low mobility networks [7].

A Shortest Multi-paths Source (SMS) [8] routing protocol based on DSR and Split Multi-paths Routing (SMR) [9] was proposed to improve efficiency of routing. The proposed protocol increased the number of selected routes between the source and the destination. Partially disjoint routes were used for routing the packets. The limitation of these 
algorithms was rapid depletion of the battery energy in the nodes along the most heavily-used paths in the communication network. Routing of packets is a significant and power consuming activity as it involves packets forwarding by many intermediate nodes. The general purpose of protocols should be to optimize energy consumption for routing for given the limited battery power in wireless nodes.

A power-aware routing protocol [10] that provides better performance in terms of link failure was proposed that finds path between the source and the destination in terms of remaining power of each node.

A power-aware routing protocol [11] based on Ad hoc On-Demand Distance Vector (AODV) protocol [12] that allows a mobile node to maintain route to a destination with more stable route selection was proposed. A faster selection of routes with minimal efforts was described.

In [10], [11] and [13], the authors take into reason the characteristic of energy of each node when a given source discovers a path to a destination. Quality of Service (QoS) requirements in terms of delay and bandwidth were not considered as in [14] and [15]. The goal of the power aware routing protocol for MANETS is to find an optimal route from a source to a destination. Route Cache plays a vital role in deciding the routes to destination. Primary Cache and Secondary caches can both be utilized for efficiency. For effective use of cache, the protocol needs to adjust to the network topology changes. Two methods to improve cache correctness and route stability were proposed in [16].

The first is the RERR-Enhance methodology in which a broken link message is sent to all nodes that contains the cached the broken link.

The second methodology is the hierarchical link cache structure accompanied with a link stability measure.

The node selects a route from the primary cache first, based on link stability, to provide stable transmission. The proposed protocol decreases the routing overhead and end to-end delay compared to typical dynamic source routing protocol. An adaptive timeout mechanism for link caches was also used.

\section{PROBLEM DEFINITION}

The objective of our proposed protocol CM-DSR is to increase the network life time by using minimum residual energy of the nodes in the MANET. This assures increase in the packet delivery ratio and to reduce the end to end delay in transmission of data packet from a given source to destination. We propose a consistent cost metric in selection of efficient route, using both the primary and secondary caches.

\section{PROPOSED WORK}

The proposed work is based on proactive DSR protocol. The protocol finds a consistent path from the multipath using the primary and secondary caches. The scheme of CM-DSR is given below.

\section{A. CM-DSR Scheme}

Step 1: During the find Route process, a minimum length path is found by comparing the length of each path stored in the primary cache and secondary cache.

Step 2: A longest path is found from the secondary cache using the length of path found from Step 1.

Step 3: From paths found from Step 1 and Step 2 an efficient path is found using the cost measure in equation 1 of the residual energy of each of the nodes in the path.

Step 4: The route that has greater value of cost measure, $\mathrm{K}$ in equation 1, shown below between multipath is selected for routing.

Step 4: The resultant path is updated in the primary cache for further routing.

\section{B. Consistent Cost Metric}

The consistent cost metric is defined as the ratio of average residual energy of the given path to the variance of the residual energy of the given path.

It is given by,

$$
\mathrm{K}=\mathrm{A} / \mathrm{V}
$$

where $A$ is the average of the given residual energy of a given path, $\mathrm{V}$ is the variance of the given residual energy of a given path.

To explain the routing scheme of CM-DSR, we consider an example with a MANET in Fig 1.

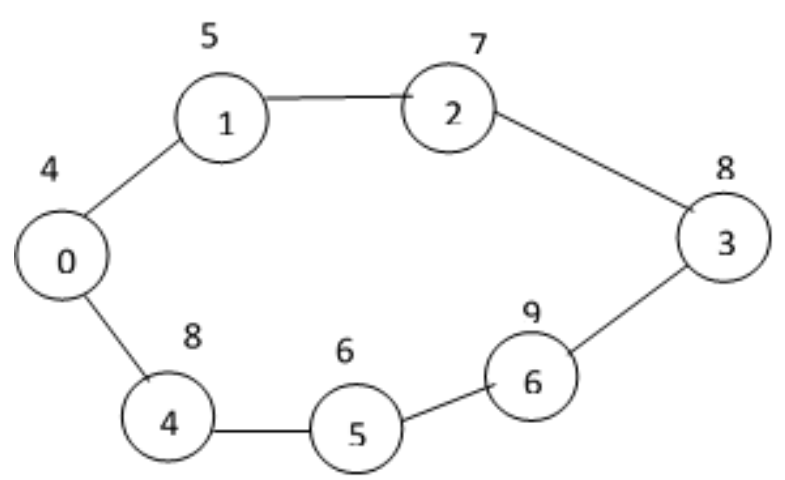

Fig 1.MANET with residual energies

In Fig 1, consider the source, $\mathrm{S}=0$ and destination, $\mathrm{D}=6$, there are two paths $0-1-2-3$ and $0-4-5-6-3$. The first path is found using Step 1 and second is found using Step 2. The residual energies values are shown above each of the nodes in the figure. Using Equation 1, the value of consistent cost metric $K$ of path $0-1-2-3$ is 0.4 Joules. The value of consistent cost metric $\mathrm{K}$ of the path is $0-4-5-6-3$ is 0.4375 . The path $0-4-5-6-3$ is selected because its consistent cost metric is greater than path $0-1-2-3$. 


\section{Performance eValuation}

\section{A. Simulation Environment}

Both DSR and CM-DSR are simulated using the NS-2 simulator. The protocols are designed in compliance with MANET protocols specified for radio/wireless models.

Table 1 , is used for assigning simulation parameters.

TABLE I: SIMULATION PARAMETERS

\begin{tabular}{ll}
\hline \hline Parameter Type & Parameter Value \\
\hline Simulator & NS-2.34 \\
Routing Protocol & DSR \\
Simulation Time (Sec) & $100 \mathrm{sec}$ \\
Simulation Area & $1000 \mathrm{~m} \mathrm{x1000} \mathrm{m}$ \\
Number of Nodes & $30-60$ \\
Transmission Range $(\mathrm{m})$ & $250 \mathrm{~m}$ \\
Channel Bandwidth & $12 \mathrm{Mbps}$ \\
Connection Rate & 5 packets/sec \\
Data Packet Size & 512 bytes \\
Traffic Source & CBR \\
Initial Energy (J) & $5-15$ \\
Transport Protocol & UDP \\
Mobile Connections & $40-60$ \\
\hline \hline
\end{tabular}

\section{B. Simulation Results}

In the Fig 2, a graph of Average Residual Energy vs. Time for 30 nodes is plotted. At 20.0s, DSR average residual energy is $300.0 \mathrm{~J}$, whereas for CM-DSR it is 320.0J.The average residual energy of CM-DSR is 6.6 percent greater than DSR. This is due to efficient average residual energy paths between given source and destination are selected for routing data packets in CM-DSR than the typical DSR protocol. At 40.0s the average residual energy of CM-DSR and DSR is same and is equal to 50.0J. Here the residual energy path between given source and destination are equal for CM-DSR and DSR. We find at any interval of time average residual energy for CM-DSR is either or equal to DSR. Thus CM-DSR performs better than DSR.

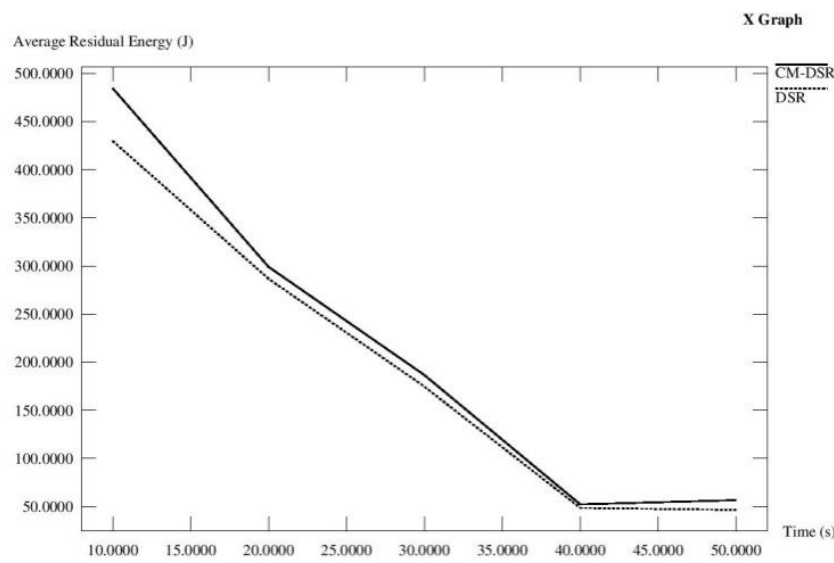

Fig.2. Average Residual Energy Vs. Time for 30 Nodes

Fig 3 is a graph of Average Residual Energy vs. Time for 40 nodes. At 20.0s, DSR average residual energy is $25.0 \mathrm{~J}$, whereas for CM-DSR it is 40.0J.The average residual energy of CM-DSR is 60 percent greater than DSR. This is due to efficient and consistent residual energy paths between given source and destination are selected for transmission in CM-DSR than classical DSR protocol at that time. At $40.0 \mathrm{~s}$ the average residual energy of DSR is $350.0 \mathrm{~J}$ and CM-DSR is 380.0J. The average residual energy of CM-DSR is 8.57 percent greater than DSR. Here also the value average residual energy of CM-DSR is greater than DSR. In the Fig 3 at any given interval of time we find average residual energy of CM-DSR is greater than DSR at given interval of time. Thus CM-DSR is more efficient than DSR. Further in Fig 2 and Fig 3 we observe at 20.0s for 30 nodes average residual energy of CM-DSR is $320.0 \mathrm{~J}$, but for 40 nodes it is 40.J. This is due the mobility of the nodes and change of topology of the network.

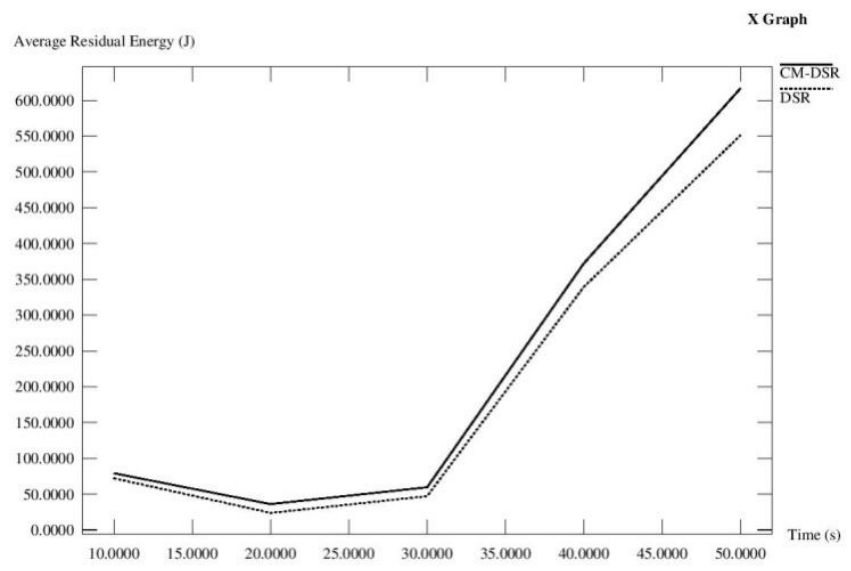

Fig.3.Average Residual Energy Vs. Time for 40 Nodes

Fig 4 is a graph of Average Transmission Energy vs. Time for 30 nodes is shown. At 25.0s, DSR average transmission energy is $1.5 \mathrm{~J}$, whereas for CM-DSR it is 1.6J.The average transmission energy of CM-DSR is 6.6 percent greater than DSR. This is due to efficient and consistent residual energy paths between given source and destination are selected for transmission in CM-DSR than DSR protocol at that time. At 40.0s the average transmission energy of DSR and CM-DSR is same and is equal to 0.6J.In the Fig 3 at any given interval of time we find average transmission energy DSR is lesser than CM-DSR. Thus CMDSR is more efficient than DSR.

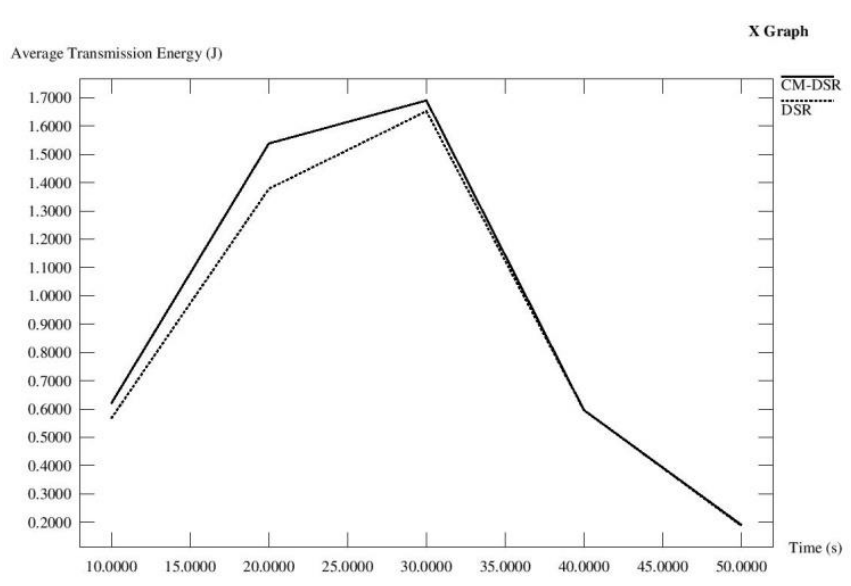

Fig.4.Average Transmission Energy Vs. Time for 30 Nodes

Fig 5 is a graph of Average Transmission Energy vs. Time for 40 nodes is given. At 30.0s average transmission energy for CM-DSR and DSR is equal and is $0.1 \mathrm{~J}$. Here the efficient average residual paths selected for given source and destination for CM-DSR and DSR are same. At 40.0s 
the average transmission energy of DSR is $2.75 \mathrm{~J}$ and CMDSR is 3.0J.Thus CM-DSR is 9.09 percent than DSR. In the Fig 5 for any given interval of time we find average transmission energy of CM-DSR is either more or equal to DSR.

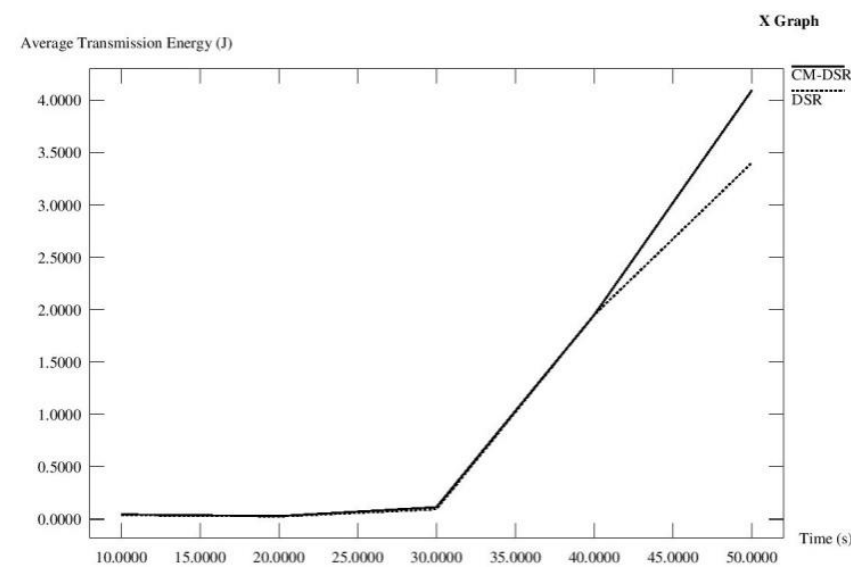

Fig.5.Average Transmission Energy Vs. Time for 40 Nodes

Fig 6 is a graph of Average Residual Energy Vs. Nodes. For 30 nodes average residual energy of DSR is $1.0 \mathrm{~J}$, whereas for CM-DSR, it is 1.1 . It is 10 percent greater than DSR. For 50 nodes the average residual energy for DSR is 4.9 J, but for CM-DSR it is $5.5 \mathrm{~J}$. Here it is 12.24 percent more than DSR. At any interval of nodes, we find average residual energy of CM-DSR is greater than or equal to DSR. Hence the performance of CM-DSR is better than DSR, due to selection of consistent cache routes

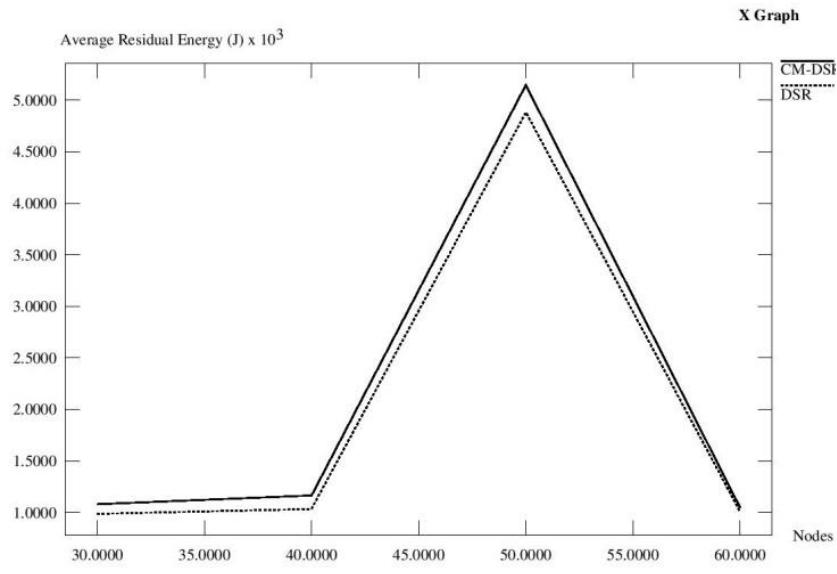

Fig.6.Average Residual Energy Vs. Nodes

Fig 7 is a graph of Average Transmission Energy Vs. Nodes. For 40 nodes average transmission energy of DSR is $5.5 \mathrm{~J}$, whereas for CM-DSR, it is 7.5. It is 36.36 percent greater than DSR. For 50 nodes the average residual energy for DSR is $44.0 \mathrm{~J}$, but for CM-DSR it is $45.5 \mathrm{~J}$. It is 3.4 percent more than DSR. At any interval of nodes in Fig 6, we find average transmission energy of CM-DSR is greater than or equal to DSR. Hence CM-DSR performs better as it selects better routes from cache.

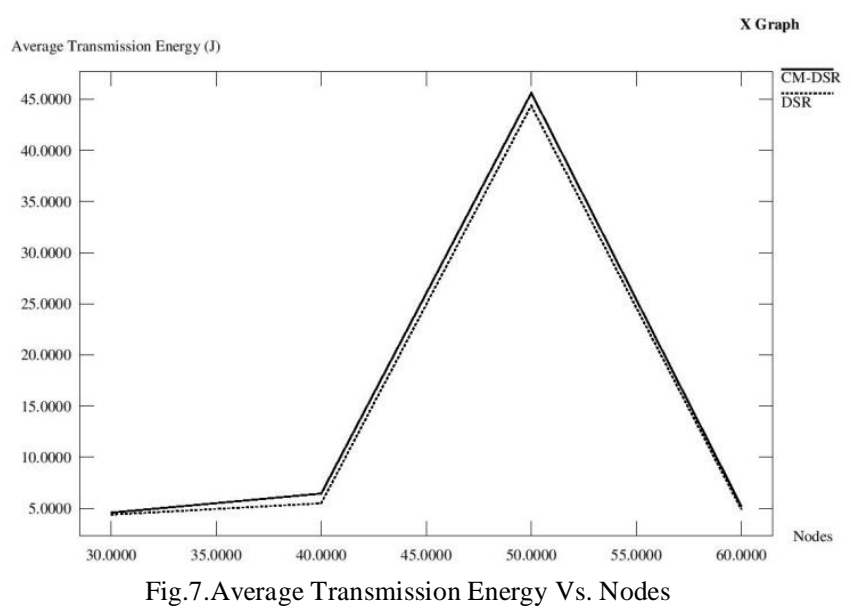

VI. CONCLUSION

Selection of efficient routes from cache for routing of data transmission is vital feature of mobile ad hoc network. Design of Energy aware protocol with proper efficient routes from primary and secondary caches can improve the performance of a protocol. We have proposed CM-DSR for MANET that uses minimum residual energy and efficiently utilizes the routes from primary and secondary caches. The simulated results show our proposed protocol performs better in terms of average residual and transmission energy per unit interval of time and density of nodes than classical DSR. Increase in average residual energy and average transmission energy improves the data transmission and reduces the delay from a given source and destination for mobile ad hoc network. In future we improve our protocol in terms of memory utilization and selection of better routes depending on the network parameters.

\section{REFERENCES}

[1] S. Das et al, "Comparative performance evaluation of routing protocols for ad hoc networks," The $7^{\text {th }}$ International Conference on Computer Communication and Networks (IC3N),Lafayette,Louisiana, USA pp 153-161,October 1998.

[2] C. Perkins and P. Bhagwat, "Highly dynamic destination -sequenced distance-vector routing (DSDV) for mobile computers," Computer Communication Review, ACM SIGCOMM, Vol. 24, No.4, ,London, England, pp. 232-244, August 1994.

[3] D. Johnson, D. Maltz, and Y.-C. Hu, "The Dynamic Source Routing for mobile ad hoc networks," IETF Internet Draft. http://www.ietf.org/internet-drafts/draft-ietf-manet-dsr-10.txt, July 2004.

[4] C. Perkins, E. Royer, and S. Das, "Ad hoc On-demand Distance Vector (AODV) Routing," RFC 3561. http://www.ietf.org/rfc/ rfc3561.txt?number=3561, July 2003.

[5] C. Perkins, E. Royer, S. Das, and M.Marina, "Performance comparison of two on-demand routing protocols for ad hoc networks," IEEE Personal Communications, Vol. 8. Issue 1, pp. 1628, February 2001.

[6] Mohammed M. "Energy efficient location aided routing protocol for wireless manets". International Journal of Computer SciTence and Information Security, 4:1-2, 2009.

[7] Toh C K. "Maximum battery life routing to support ubiquitous mobile computing in wireless ad hoc networks". Master's thesis, IEEE Communications Magazine, June 2001.

[8] H.Zafar, D.Harle, I.Andonovic, and Mahmood Ashraf, "Performance Evaluation of Shortest Multipath Source Routing Scheme”, IET communications, Vol. 3, No.5, May 2009.

[9] D. Johnson, Y. Hu and D. Maltz, "The Dynamic Source Routing Protocol for Mobile Ad-hoc Networks for IPv4", RFC 4728, Feb.2007. 
[10] V.Kumar, R. Shrivastava and V.Malik, "An Algorithm for Improvement of Link Failure in Mobile Ad-Hoc Networks," International Journal for Science and Emerging Technologies with Latest Trends, 2013.

[11] D.Sharma, A. Kush, "Power Aware Routing Scheme in Ad Hoc Networks," JOURNAL OF COMPUTING, 2011.

[12] C. Perkins, E. Belding-Royer, " Ad Hoc On-demand Distance Vector (AODV) Routing, RFC, 2003.

[13] S.K.Dhurandher, S.Misra, M.S.Oubaidat, V.Bansal, P.R.Singh and V.Punia, "An energy-efficient ad hoc on-demand routing protocol for mobile ad hoc networks", International Journal of Communication System", 2009.

[14] L.Chen and B. Heinzelman, "QoS-aware routing based on bandwidth estimation for mobile Ad Hoc networks", IEEE Journal on Selected Areas in Communications, 2005.

[15] H.Zafar, D.Harle, I.Andonovic, and Mahmood Ashraf, "Performance Evaluation of Shortest Multipath Source RoutingScheme",IET communications, Vol. 3, No.5, May 2009.

[16] Tsung- Chuan Huang, Chi-Chen Chan Kaohsiung, Taiwan "Caching Strategies for Dynamic Source Routing in Mobile Ad Hoc Networks" IEEE Communications Society, WCNC 2007 pp.4243-4246. 